\title{
Que princípios éticos devem definir o estabelecimento de prioridades entre doentes?
}

Which Ethical Principles Should Define Prioritization of Patients?

Quels sont les principes éthiques qui doivent définir la mise en place de priorités entre les malades?

\section{Micaela Pinho e Ana Pinto Borges}

\section{OpenEdition}

\section{Journals}

Edição electrónica

URL: http://journals.openedition.org/rccs/6681

DOI: $10.4000 /$ rccs.6681

ISSN: 2182-7435

\section{Editora}

Centro de Estudos Sociais da Universidade de Coimbra

Edição impressa

Data de publição: 1 Setembro 2017

Paginação: 129-148

ISSN: 0254-1106

\section{Refêrencia eletrónica}

Micaela Pinho e Ana Pinto Borges, « Que princípios éticos devem definir o estabelecimento de prioridades entre doentes? », Revista Crítica de Ciências Sociais [Online], 113 | 2017, colocado online no dia 27 julho 2017, criado a 19 abril 2019. URL : http://journals.openedition.org/rccs/6681 ; DOI : $10.4000 /$ rccs. 6681

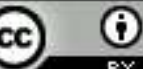




\title{
MICAELA PINHO, ANA PINTO BORGES
}

\section{Que princípios éticos devem definir o estabelecimento de prioridades entre doentes?}

\begin{abstract}
Este artigo visa investigar e comparar os julgamentos de valor de estudantes universitários portugueses da área empresarial e da saúde em matéria de priorização de doentes e comparar estes julgamentos, indiretamente, com os da população inglesa. Para tal, recorre-se a uma abordagem quali-quantitativa aplicada a um exercício hipotético de racionamento que envolve a priorização de quatro doentes, distinguidos pelas suas características pessoais e condições de saúde. Os dados foram tratados através de análises bivariadas e de conteúdo. Conclui-se que os estudantes portugueses suportam um pluralismo de princípios éticos similares aos da sociedade inglesa. Contudo, poderá existir entre os estudantes portugueses um conflito de opiniões no que respeita à priorização dos doentes mais novos.
\end{abstract}

Palavras-chave: cuidados de saúde; gestão hospitalar; priorização de doentes; princípios éticos; Portugal.

\section{Introdução}

Em muitos países desenvolvidos, a questão do racionamento dos cuidados de saúde, particularmente ao nível micro, tem sido encarada como tabu e a sua discussão evitada pelos líderes políticos. Os responsáveis pelas políticas de saúde preferem falar em reformas estruturais de combate às ineficiências, ao mesmo tempo que delegam nos profissionais de saúde a responsabilidade última de prosseguir um racionamento de tipo discricionário. Lidar com a escassez de recursos de forma implícita começa, porém, a ser cada vez menos uma opção. Por um lado, os profissionais de saúde, sobretudo os médicos, permanentemente sujeitos ao juízo da opinião pública, começam a sentir a necessidade de partilhar a responsabilidade das decisões de microalocação com os políticos e gestores hospitalares, responsáveis últimos pelo dimensionamento dos recursos que lhes cabe racionalizar (Reys, 1991). Por outro lado, o advento da crise financeira internacional de 2008, ao limitar fortemente o financiamento de muitos sistemas de saúde (Reeves e McKee, 2015), aumentou 
a pressão social para a transparência das decisões. Conferir ao racionamento um carácter explícito implica definir de forma clara os critérios que devem dominar as decisões sobre que doente(s) tratar. Nos últimos anos assistiu-se a um proliferar de artigos na literatura com propostas de princípios éticos que fundamentam o estabelecimento de prioridades entre doentes (Beauchamp e Childress, 2001; Broome, 1984; Butler, 1999; Clark e Weale, 2012; Persad et al., 2009; Powers e Faden, 2008; Williams e Cookson, 2000). Adicionalmente, existe uma crescente literatura empírica que avalia e compara a opinião dos diferentes atores sociais sobre os critérios que devem nortear a priorização de doentes (Cookson e Dolan, 1999; Diederich e Salzmann, 2015; Exel et al., 2015; Gagnon et al., 2011; Kaplan e Baron-Epel, 2013; Kasemsup et al., 2008; Lees et al., 2002; Strech et al., 2009; Winkelhage et al., 2013). Os resultados destes estudos são, porém, heterogéneos e multifacetados. Esta variabilidade resulta, em grande parte, do facto de os estudos usarem diferentes metodologias e diferentes desenhos das questões, o que dificulta a comparabilidade entre eles e não permite que sejam retiradas conclusões sólidas. Tem-se constatado que pequenas alterações no contexto (framing) das questões podem alterar dramaticamente as preferências declaradas pelos respondentes (Rabin, 1998). Uma vasta literatura na área da psicologia mostra que as pessoas não têm preferências bem definidas. Estas tendem antes a ser construídas durante o processo de eliciação a partir de princípios heurísticos que simplificam as questões (Kahneman e Tversky, 1979). O presente artigo pretende colmatar esta limitação fazendo uso de um desenho previamente aplicado em Inglaterra, país com um sistema de saúde semelhante ao português.

Este estudo revela-se oportuno na medida em que a explicitação das medidas de racionamento começa a ganhar proeminência política em Portugal como decorrência do processo de consolidação orçamental. Um primeiro avanço nesta matéria encontra-se plasmado no parecer emitido pelo Conselho Nacional de Ética para as Ciências da Vida sobre a existência de fundamentos éticos para a prossecução de medidas de contenção de custos com medicamentos (CNECV, 2012). Acresce ainda que, a nível institucional, desde 2002 a "empresarialização" da saúde tem vindo a ganhar relevo no sistema de saúde português, e mais concretamente no SNS. Os Hospitais Empresa Pública Empresarial foram, a partir de 2005, a concretização da introdução de novos mecanismos de gestão privada nos hospitais públicos (Decreto-Lei n. ${ }^{\circ} 233 / 2005$ de 29 de dezembro). A gestão destas unidades está a cargo de equipas multidisciplinares compostas por economistas/gestores e profissionais de saúde que, em momentos de maior contenção financeira, podem revelar opiniões conflituantes. Neste contexto, parece importante conhecer os julgamentos de valor defendidos por cada um destes atores sociais, por estarem diretamente envolvidos nas decisões de racionamento. 
O presente estudo recorre a uma abordagem quali-quantitativa suportada em cenários hipotéticos de racionamento previamente desenvolvidos e aplicados (Cookson e Dolan, 1999). O estudo foi organizado de acordo com dois objetivos principais. Primeiramente, explorar se os julgamentos de valor expressos por estudantes universitários das áreas empresariais em relação à priorização de doentes são similares aos defendidos pelos estudantes das áreas da saúde. Esta comparação permite-nos avaliar a existência de eventuais conflitos de opiniões entre membros de equipas com responsabilidade nas administrações hospitalares. Em segundo lugar, e dada a similaridade dos cenários de racionamento, pretendemos comparar indiretamente a opinião dos estudantes portugueses com a dos membros da sociedade inglesa.

\section{Princípios de justiça substantivos de microalocação dos recursos}

Os princípios de justiça substantivos mais discutidos na literatura defendem que as prioridades entre doentes devem ser estabelecidas de acordo com: a) a maximização dos ganhos em saúde (Culyer, 1997); b) a necessidade (Mooney, 1994); c) a redução das desigualdades em saúde (LeGrand, 1982; Rawls, 1999; Williams, 1997) e d) a lotaria (Childress, 1983).

Ainda que todos estes critérios sejam legítimos e defensáveis no campo da justiça distributiva, a sua natureza multidimensional pode redundar em diferentes interpretações do que se entende por uma afetação justa dos recursos na saúde. Neste sentido, segue-se uma breve descrição de cada princípio.

O princípio da maximização dos ganhos de saúde sustenta-se no critério utilitarista e defende que uma alocação ótima dos recursos é a que garante a maximização da saúde agregada da população, por unidade de custo. Neste sentido, merece prioridade o doente que apresenta melhor prognóstico. Este princípio extravasa os ganhos individuais para incorporar as consequências indiretas da saúde do doente. São valorados aspetos como o estatuto laboral do doente e/ou a sua capacidade contributiva.

O princípio da necessidade surge em oposição ao racionamento pelo preço e, ainda que bem aceite, a sua aplicação esbarra na dificuldade em definir "necessidade". Necessidade em saúde pode ser avaliada em termos técnicos ou sociais. Uma visão técnica equipara necessidade a capacidade de benefício (Culyer e Wagstaff, 1993). Neste sentido, necessidade de cuidados de saúde existe apenas e só se (ou enquanto) o doente puder beneficiar desses cuidados. Esta interpretação constitui uma variante do princípio mais geral da alocação de acordo com a maximização dos ganhos de saúde. Necessidade em saúde pode ainda ser, tecnicamente, equiparada a gravidade do estado de saúde. A necessidade clínica avalia a gravidade, por exemplo, em termos de dor/sofrimento. Em casos extremos de vida ou morte, 
a literatura identifica o princípio do resgate (rule of rescue; Hadorn, 1991) que sustenta que a sociedade tem o dever ético de fazer todos os possíveis por ajudar aqueles que enfrentam um risco imediato de morte. A avaliação social de necessidade encerra uma dimensão emocional, ao interceder pela vulnerabilidade ou fragilidade económica e/ou afetiva do doente e pela sua responsabilidade social (Fortes e Zoboli, 2002).

O princípio da redução das desigualdades em saúde tem-se revelado controverso sobretudo pela falta de consenso em definir igualdade em saúde. Um princípio igualitário amplamente discutido sustenta a igualdade na saúde auferida ao longo da vida - equidade intergeracional. De acordo com o fair-innings (Williams, 1997) todos os indivíduos têm direito a uma vida longa e com qualidade. Merece prioridade o doente mais novo, por ser aquele que se encontra mais longe de alcançar este desiderato. A evidência empírica parece sugerir que a ideia de priorizar os doentes mais novos é praticamente consensual em todas as culturas (Pinho, 2014). Porém, quase nunca é possível concluir se as preferências pelo doente mais novo são justificadas pelo facto de este ter vivido menos ou, antes, por ser expectável que tenha uma maior capacidade de benefício. A primeira explicação insere-se no argumento equitativo do fair-innings, enquanto a segunda é consistente com o critério da eficiência refletido no princípio da maximização da saúde esperada. Esta dupla explicação para a preferência pelos doentes mais novos mostra a importância de conhecer as razões das sociedades pela priorização dos doentes. Outra variante do princípio da redução das desigualdades em saúde contesta a ideia da igualização da saúde entre todos para defender a igualdade de oportunidades substantivas (LeGrand, 1982; Roemer, 1998). Este princípio deriva da teoria mais geral de Amartya Sen. Para Sen (1980), desde que a todos os indivíduos seja garantida a mesma oportunidade de usufruir de uma vida saudável, qualquer desigualdade na saúde que venha a ocorrer será atribuída à liberdade de escolha individual e, consequentemente, considerada justa. O choicism (Cookson e Dolan, 1999) constitui uma noção implícita de justiça, ao definir a prioridade de acesso aos cuidados de saúde em função do merecimento ou responsabilização moral do indivíduo pela sua saúde. A Constituição da República Portuguesa, no n. ${ }^{\circ} 1$ do artigo 64 , alude a este critério ao referir que "todos têm direito à proteção da saúde e o dever de a defender e promover" (Constituição da República Portuguesa, 2005).

Por fim, decidir que doente tratar pode ser considerado não ético, ou tomar essa decisão pode revelar-se moralmente intolerável e fonte de "desutilidade" (Coast, 2000). O recurso à lista de espera (ordem de chegada) ou à randomização são procedimentos que dispensam qualquer avaliação pessoal do doente (Childress, 1983; Beauchamp e Childress, 2001). 


\section{Metodologia}

O presente estudo combina dados quantitativos e qualitativos, com o propósito de explorar e comparar os princípios éticos suportados por dois grupos de estudantes universitários.

\section{Descrição da amostra}

Um questionário foi aplicado, durante o ano de 2014, a uma amostra aleatória de 177 estudantes universitários (inscritos no último ano do ciclo de estudos de licenciatura) de instituições públicas e privadas localizadas no norte e centro de Portugal. A amostra inclui estudantes das áreas científicas das ciências empresariais: 70 estudantes de economia e 45 estudantes de gestão. Das ciências da saúde inclui: 36 estudantes de medicina e 26 estudantes de psicologia clínica. Elegemos estudar estes dois grupos por acreditarmos que a atitude dos estudantes pode, cautelosamente (devido à falta de experiência profissional), ser usada como indicador do parecer dos correspondentes profissionais (e potenciais atores sociais). Assim, importa questionar se os estudantes das áreas empresariais são, pela sua formação, mais adeptos da prossecução da eficiência que os colegas das ciências da saúde. De forma análoga, importa saber se os estudantes da área da saúde apoiam mais o princípio da alocação de acordo com a necessidade clínica que os demais colegas de economia/gestão. Da análise descritiva da amostra é possível concluir que, a maioria dos respondentes, têm entre 20 e 35 anos (56\%) e que existe uma proporcionalidade de género, com $54 \%$ dos respondentes do sexo feminino. Os inquiridos são, na sua maioria, solteiros (86\%) e dispõem de um rendimento mensal (agregado familiar) entre 851 e $2500 €$. Os estudantes residem, maioritariamente, em centros não urbanos ( $54 \%$ ). Relativamente à perceção do seu estado de saúde, a quase totalidade dos respondentes $(92 \%)$ considera ter uma saúde boa ou muito boa e a maioria (55\%) diz não sofrer nem nunca ter sofrido (nem ninguém do seu agregado familiar) de qualquer doença grave nos últimos 5 anos.

\section{Descrição do questionário}

Os dados foram recolhidos através de um questionário. O questionário contemplou um exercício hipotético de racionamento semelhante a um previamente desenhado (Cookson e Dolan, 1999). O exercício consiste em estabelecer uma ordem de prioridade de atendimento de quatro doentes que se distinguem pelas suas características pessoais e de saúde. A Tabela 1 descreve os cenários apresentados.

O questionário incluiu um espaço para os respondentes exporem as razões para a ordenação feita. Os inquiridos foram informados do carácter hipotético 
e não real deste exercício. Os autores do estudo seguiram as orientações de Dolan et al. (1999), concedendo aos estudantes tempo suficiente para formularem decisões ponderadas e refletidas. Conscientes de que assuntos desta natureza são muito sensíveis, os autores do estudo evitaram manter contacto com os inquiridos enquanto estes preenchiam o inquérito. Foi ainda garantido aos respondentes total anonimato. Com estas preocupações, procurou acautelar-se quaisquer relutâncias que os estudantes pudessem sentir em emitir as suas verdadeiras opiniões. A participação dos estudantes foi voluntária. Os participantes autorizaram o tratamento dos dados.

Embora os cenários usados neste estudo sejam muito semelhantes aos aplicados em Inglaterra (Cookson e Dolan, 1999), eles divergem em alguns pontos, como: a) uso de questionário versus focus groups; b) pequenas diferenças nas características de saúde dos doentes; c) remoção das fotografias dos doentes e d) diferenças na amostra - estudantes universitários versus população, em geral. Por esta razão não é possível fazer uma comparação robusta dos resultados dos dois estudos, limitando-nos a uma comparação indireta.

\section{TABELA 1 - Cenários do exercício hipotético de priorização de doentes}

\begin{tabular}{|c|c|}
\hline Como escolheria? & $\begin{array}{l}\text { Paulo tem } 18 \text { anos e foi atropelado, do que resultaram } \\
\text { múltiplos ferimentos faciais e danos psicológicos. } \\
\text { Uma cirurgia plástica corrige os danos faciais. }\end{array}$ \\
\hline $\begin{array}{l}\text { Suponha que é decisor } \\
\text { numa unidade hospitalar } \\
\text { onde dispõe de recursos } \\
\text { que lhe permitem } \\
\text { tratar um dos seguintes } \\
\text { doentes. }\end{array}$ & $\begin{array}{l}\text { Fernanda tem } 45 \text { anos e é solteira. Fernanda sofre de obesidade, } \\
\text { como resultado de longos anos de uma alimentação inadequada } \\
\text { à base de fast-food e de um modo de vida sedentário. Fernanda } \\
\text { há } 2 \text { anos que tem levado uma alimentação mais saudável. } \\
\text { Existe um tratamento disponível, com } 75 \% \text { de eficácia que lhe } \\
\text { proporcionará mais anos de vida com qualidade. }\end{array}$ \\
\hline \multirow{2}{*}{ 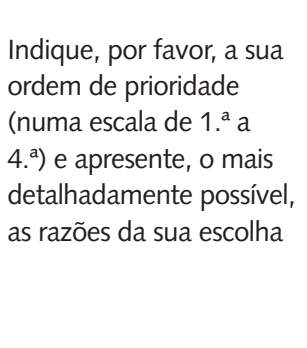 } & $\begin{array}{l}\text { Rosa tem } 65 \text { anos e está quase cega. Ela aguarda há } 3 \text { anos por } \\
\text { uma cirurgia que lhe removerá as cataratas. A sua visão tem } \\
\text { piorado ao longo do tempo e em breve deixará de conseguir } \\
\text { viver sozinha. Ela não tem família. A operação permitir-lhe-á ser } \\
\text { independente. }\end{array}$ \\
\hline & $\begin{array}{l}\text { Miguel tem } 8 \text { anos e uma leucemia. Ele tem uma probabilidade } \\
\text { de sobrevivência de } 50 \% \text {. Existe porém disponível um novo } \\
\text { tratamento que foi parcialmente testado num número reduzido } \\
\text { de casos. }\end{array}$ \\
\hline
\end{tabular}

\section{Análises}

A análise quantitativa dos dados foi prosseguida através do software SPSS (versão 22). Foi usada uma análise descritiva para sumariar a ordenação feita dos doentes e aplicado o teste Mann-Whitney para avaliar a existência de diferenças 
em cada lugar da ordenação dos doentes e a área de formação científica dos respondentes. O teste Mann-Whitney, assim como o teste Kruskal-Wallis foram, ainda, usados para testar a relação entre os princípios distributivos e as características sociodemográficas e de estado de saúde dos respondentes. Por fim, foi aplicado o teste Kruskal-Wallis para explorar a influência dos princípios distributivos na escolha do primeiro doente a tratar. A análise qualitativa avaliou as justificativas apresentadas pelos respondentes para cada ordenação feita dos doentes de acordo com a abordagem de conteúdo de Bardin (2013). As razões expostas pelos respondentes foram identificadas e classificadas em categorias e subcategorias. Procedeu-se a uma codificação de grupos de palavras usando um esquema de classificação interativo. As ideias foram classificadas em categorias gerais correspondentes aos princípios de racionamento identificados na literatura (descritos anteriormente). As razões referenciadas pelos respondentes foram codificadas em "Princípios" (categoria principal) e "Fatores" (subcategoria). Os princípios correspondem aos critérios gerais de distribuição de cuidados de saúde, enquanto os fatores retratam interpretações de cada princípio distributivo e, consequentemente, os aspetos específicos da tomada de decisões. A identificação e codificação das categorias foram realizadas pela investigadora principal do estudo (primeira autora).

\section{Resultados}

\section{Resultados quantitativos - Estabelecimento de prioridades entre doentes}

A Tabela 2 sintetiza os resultados do exercício de ordenação dos quatro doentes realizado pelos dois grupos de estudantes e apresenta os resultados do teste que avalia as diferenças na ordenação feita entre eles (Mann-Whitney). As diferenças com significância estatística são assinaladas com asterisco (*).

Os resultados mostram que, do total de 177 inquiridos, apenas 3 (2\%) se recusaram a ordenar os doentes por prioridade de atendimento. Nenhum respondente colocou na mesma ordenação mais do que um doente. O Miguel foi o doente escolhido para ser atendido em primeiro lugar pela maioria dos inquiridos $(72 \%)$. A doente Rosa foi escolhida para ser tratada em segundo lugar por $38 \%$ dos inquiridos. O terceiro lugar de atendimento foi atribuído ao Paulo por $32 \%$ dos estudantes inquiridos. A Fernanda, foi colocada em última prioridade por $51 \%$ dos respondentes. Esta ordenação converge para os resultados do estudo original (Cookson e Dolan, 1999). Ainda assim, foram mais os respondentes ingleses a conferir prioridade de atendimento à criança $(80 \%)$, relativamente menos os que colocaram a doente idosa em segundo lugar $(31,7 \%)$, e mais os que atribuíram ao jovem adulto a terceira prioridade $(41,7 \%)$. Um igual padrão de preferência foi registado entre portugueses 
e ingleses na concessão da última posição de atendimento à Fernanda ( $51 \%$ e $51,7 \%$ entre os portugueses e ingleses, respetivamente).

Analisando a ordenação dos doentes por área de formação científica dos estudantes inquiridos, constata-se que apenas existem diferenças estatisticamente significativas para o doente a atender em terceiro lugar (teste Mann-Whitney, $\mathrm{z}=2864,500, \mathrm{p}=0.025$ ). Os estudantes das ciências empresariais nomeiam o Paulo para ser tratado em terceiro lugar, enquanto os das ciências da saúde preferem que este lugar seja ocupado pela Fernanda.

TABELA 2 - Ordenação dos doentes por grupo de estudantes

\begin{tabular}{|c|c|c|c|c|}
\hline & $\begin{array}{c}\text { Ciências } \\
\text { Empresariais } \\
(n=115)\end{array}$ & $\begin{array}{l}\text { Ciências } \\
\text { Saúde } \\
(n=62)\end{array}$ & $\begin{array}{l}\text { Total } \\
(\%)\end{array}$ & $\begin{array}{c}\text { Teste } \\
\text { Mann-Whitney } \\
\text { (p-value) }\end{array}$ \\
\hline \multicolumn{5}{|l|}{ Quem tratar em $1 .^{\circ}$ lugar? } \\
\hline Paulo & $16(14 \%)$ & $7(11 \%)$ & $23(13 \%)$ & \multirow{5}{*}{$\begin{array}{r}3523,000 \\
(0,870)\end{array}$} \\
\hline Fernanda & $2(2 \%)$ & $2(3 \%)$ & $4(2 \%)$ & \\
\hline Rosa & $12(10 \%)$ & $8(13 \%)$ & $20(11 \%)$ & \\
\hline Miguel & $84(73 \%)$ & $43(70 \%)$ & $127(72 \%)$ & \\
\hline Não sabe/Não responde & $1(1 \%)$ & $2(3 \%)$ & $3(2 \%)$ & \\
\hline \multicolumn{5}{|l|}{ Quem tratar em $2 .^{\circ}$ lugar? } \\
\hline Paulo & $38(33 \%)$ & $19(31 \%)$ & $57(32 \%)$ & \multirow{5}{*}{$\begin{array}{r}3504,500 \\
(0,845)\end{array}$} \\
\hline Fernanda & $14(12 \%)$ & $10(16 \%)$ & $24(14 \%)$ & \\
\hline Rosa & $43(37 \%)$ & $25(40 \%)$ & $68(38 \%)$ & \\
\hline Miguel & $19(17 \%)$ & $6(10 \%)$ & $25(14 \%)$ & \\
\hline Não sabe/Não responde & $1(1 \%)$ & $2(3 \%)$ & $3(2 \%)$ & \\
\hline \multicolumn{5}{|l|}{ Quem tratar em 3. lugar? } \\
\hline Paulo & $45(39 \%)$ & $11(18 \%)$ & $56(32 \%)$ & \multirow{5}{*}{$\begin{array}{r}2864,500 * \\
(0,025)\end{array}$} \\
\hline Fernanda & $26(23 \%)$ & $24(39 \%)$ & $50(28 \%)$ & \\
\hline Rosa & $36(31 \%)$ & $17(27 \%)$ & $53(30 \%)$ & \\
\hline Miguel & $7(6 \%)$ & $8(13 \%)$ & $15(8 \%)$ & \\
\hline Não sabe/Não responde & $1(1 \%)$ & $2(3 \%)$ & $3(2 \%)$ & \\
\hline \multicolumn{5}{|l|}{ Quem tratar em $4 .^{\circ}$ lugar? } \\
\hline Paulo & $21(18 \%)$ & $23(37 \%)$ & $44(25 \%)$ & \multirow{5}{*}{$\begin{array}{r}3111,000 \\
(0,128)\end{array}$} \\
\hline Fernanda & $67(58 \%)$ & $24(39 \%)$ & $91(51 \%)$ & \\
\hline Rosa & $23(20 \%)$ & $10(16 \%)$ & $33(19 \%)$ & \\
\hline Miguel & $3(3 \%)$ & $3(5 \%)$ & $6(3 \%)$ & \\
\hline Não sabe/Não responde & $1(1 \%)$ & $2(3 \%)$ & $3(2 \%)$ & \\
\hline $\mathrm{N}$ & $115(100 \%)$ & $62(100 \%)$ & 177 & \\
\hline
\end{tabular}




\section{Resultados qualitativos - Justificativas para a priorização dos doentes}

A Tabela 3 sumaria o número de vezes que cada princípio distributivo foi invocado pelos dois grupos de estudantes e transcreve as razões mais vezes referidas.

\section{TABELA 3 - Princípios éticos e justificações mencionados pelos dois grupos de estudantes}

\begin{tabular}{lccc}
\hline & Ciências & Ciências & \\
Princípios & Fatores & Empresariais & Saúde \\
& $(n=115)$ & $(n=62)$ & Justificativas \\
& & & \\
\hline
\end{tabular}

P1.

Lotaria/Ordem

Igual
prioridade

1
$(0,9 \%)$

2
$(3,2 \%)$

1
$(1,6 \%)$
"Ninguém tem o direito de tomar estas decisões"

Chegada

“Paulo beneficia durante

\section{Maximizar}

P2. ganhos anos/

Maximização

Saúde

\begin{tabular}{|c|c|c|c|c|}
\hline Saúde & $\begin{array}{l}\text { Contributo } \\
\text { económico }\end{array}$ & $\begin{array}{r}6 \\
(5,2 \%)\end{array}$ & $\begin{array}{r}2 \\
(3,2 \%)\end{array}$ & $\begin{array}{l}\text { "Paulo pode contribuir } \\
\text { para a economia" } \\
\text { "Miguel será um futuro } \\
\text { contribuinte" }\end{array}$ \\
\hline $\begin{array}{l}\text { P3. } \\
\text { Necessidade } \\
\text { = Capacidade } \\
\text { Benefício }\end{array}$ & $\begin{array}{l}\text { Eficácia } \\
\text { tratamento }\end{array}$ & $\begin{array}{r}10 \\
(8,7 \%)\end{array}$ & $\begin{array}{r}6 \\
(9,7 \%)\end{array}$ & $\begin{array}{l}\text { "Paulo e Rosa apresentam } \\
\text { maior capacidade } \\
\text { recuperação e/ou de ganhar } \\
\text { mais qualidade vida" } \\
\text { "Paulo fica completamente } \\
\text { curado" } \\
\text { "A Fernanda tem 75\% } \\
\text { de probabilidade de ficar } \\
\text { curada" }\end{array}$ \\
\hline \multirow{3}{*}{$\begin{array}{l}\text { P4. } \\
\text { Necessidade } \\
\text { clínica }\end{array}$} & Urgência & $\begin{array}{r}16 \\
(13,9 \%)\end{array}$ & $\begin{array}{r}6 \\
(9,7 \%)\end{array}$ & \multirow{3}{*}{$\begin{array}{l}\text { "Miguel e Rosa são os } \\
\text { doentes mais urgentes" } \\
\text { de miguel corre o risco } \\
\text { de morrer" } \\
\text { na aparência físicos } \\
\text { podem estar a causar-lhe } \\
\text { sofrimento psicológico" }\end{array}$} \\
\hline & Dor/sofrimento & $\begin{array}{r}5 \\
(4,3 \%)\end{array}$ & $\begin{array}{r}1 \\
(1,6 \%)\end{array}$ & \\
\hline & Eminência morte & $\begin{array}{r}10 \\
(8,7 \%)\end{array}$ & $\begin{array}{r}2 \\
(3,2 \%)\end{array}$ & \\
\hline
\end{tabular}
mais tempo do tratamento"

"Miguel por ser mais novo pode beneficiar durante mais anos do tratamento" 
cont.

\begin{tabular}{|c|c|c|c|c|}
\hline Princípios & Fatores & $\begin{array}{c}\text { Ciências } \\
\text { Empresariais } \\
(n=115)\end{array}$ & $\begin{array}{l}\text { Ciências } \\
\text { Saúde } \\
(n=62)\end{array}$ & Justificativas \\
\hline & $\begin{array}{c}\text { Fragilidade } \\
\text { Vulnerabilidade }\end{array}$ & $\begin{array}{r}6 \\
(5,2 \%)\end{array}$ & $\begin{array}{r}3 \\
(4,8 \%)\end{array}$ & \multirow{2}{*}{$\begin{array}{l}\text { "Miguel e Rosa são os mais } \\
\text { dependentes" } \\
\text { "Rosa ficará, em breve, } \\
\text { dependente" } \\
\text { "Miguel porque nenhum pai/ } \\
\text { mãe merece passar pela dor } \\
\text { de perder um filho" }\end{array}$} \\
\hline $\begin{array}{l}\text { P5. } \\
\text { Necessidade } \\
\text { Social }\end{array}$ & $\begin{array}{c}\text { Ameaça } \\
\text { Independência }\end{array}$ & $\begin{array}{r}7 \\
(6,1 \%)\end{array}$ & $\begin{array}{r}5 \\
(8,1 \%)\end{array}$ & \\
\hline $\begin{array}{l}\text { P6. } \\
\text { Igualdade } \\
\text { Saúde }\end{array}$ & Fair-innings & $\begin{array}{r}61 \\
(53,0 \%)\end{array}$ & $\begin{array}{r}18 \\
(29,0 \%)\end{array}$ & $\begin{array}{l}\text { "Miguel é uma criança com } \\
\text { uma vida pela frente" } \\
\text { "Miguel e Paulo são os mais } \\
\text { novos e merecem viver } \\
\text { a vida" }\end{array}$ \\
\hline $\begin{array}{l}\text { P7. } \\
\text { Choicism }\end{array}$ & $\begin{array}{c}\text { Penalizar } \\
\text { Comportamentos } \\
\text { Risco }\end{array}$ & $\begin{array}{r}1 \\
(0,9 \%)\end{array}$ & $\begin{array}{r}0 \\
(0,0 \%)\end{array}$ & $\begin{array}{l}\text { "Fernanda sabia que estava } \\
\text { a prejudicar a saúde" }\end{array}$ \\
\hline
\end{tabular}

O levantamento geral das justificativas mostra que se inserem nos critérios distributivos propostos na literatura e anteriormente descritos. Os resultados reforçam o reduzido apoio conferido ao princípio da lotaria ou ordem de chegada. Destaca-se, ainda, a pouca relevância conferida à penalização dos comportamentos de risco, referenciada apenas pelos estudantes das ciências empresariais. Os respondentes ingleses também revelaram pouca concordância com a ideia de priorizar doentes com base no choicism (Cookson e Dolan, 1999). O princípio da equidade intergeracional foi aquele que mais adesão recebeu por parte dos dois grupos de inquiridos. Ainda assim, esta preferência foi mais significativa nos estudantes da área empresarial $(53 \%$ contra apenas $29 \%$ dos estudantes das ciências da saúde). A razão mais invocada foi a de que o "Miguel e Paulo são os doentes mais jovens, e como tal têm uma vida pela frente".

A necessidade clínica, na vertente da gravidade do estado de saúde, foi o segundo critério mais mencionado pelos estudantes da área empresarial $(13,9 \%)$ seguido da necessidade enquanto capacidade de benefício $(8,7 \%)$. Os estudantes com formação em saúde parecem valorar de igual modo a capacidade de benefício e a gravidade do estado de saúde. Este grupo de inquiridos suporta, ainda assim, mais a eficácia clínica dos tratamentos que os seus colegas da área das ciências empresariais. Os estudantes da área da saúde parecem mais interessados numa análise factual do prognóstico 
clínico do que os colegas das ciências empresariais. Este resultado sugere que os estudantes das ciências da saúde podem vir a confrontar-se com um potencial conflito entre o critério da eficiência (maximização dos ganhos de saúde) e o da equidade (argumento do fair-innings). Este dilema poderá evidenciar-se perante uma decisão que envolva uma criança com um prognóstico reservado. Relativamente ao critério da maximização da saúde os estudantes, sobretudo da área das ciências empresariais, invocaram o potencial contributo económico dos doentes. Neste contexto, foi dada prioridade ao Paulo e ao Miguel por serem considerados potenciais contribuintes.

Estes resultados qualitativos podem constituir uma explicação para a diferença de ordenação dos doentes (Tabela 2). O Paulo (jovem adulto) foi colocado em terceira prioridade pelos estudantes da área empresarial provavelmente por causa do seu maior contributo económico para o país. Os estudantes da área da saúde poderão ter escolhido a Fernanda por serem menos penalizadores dos comportamentos individuais. Estas interpretações, por serem especulativas, carecem de mais pesquisa.

A dimensão emocional de necessidade é evidenciada pelos dois grupos de inquiridos com a ameaça de independência a ser o fator mais vezes citado para justificar o atendimento prioritário da doente idosa e em risco de ficar cega.

Em síntese, os resultados indicam que em termos de princípios gerais de justiça os estudantes inquiridos parecem adotar uma posição pluralista, ao privilegiarem três dos sete princípios descritos: a) igualdade na saúde através da equidade intergeracional ou fair-innings; b) necessidade clínica na sua vertente da eminência de morte ou rule-of-rescue e c) necessidade técnica na vertente da prossecução da eficiência ao ponderar o resultado do tratamento.

Os membros da população inglesa privilegiaram os mesmos três princípios, mas conferiram-lhes uma importância distinta. O critério da necessidade clínica foi considerado o mais importante, seguido do critério da maximização dos ganhos em saúde e da equidade intergeracional (Cookson e Dolan, 1999).

Para explorar se as características sociodemográficas e de saúde dos inquiridos foram determinantes na importância atribuída a cada princípio distributivo, foram aplicados os testes de Mann-Whitney e Kruskal-Wallis. Os resultados apresentados na Tabela 4 mostram que apenas a área científica se revelou significativa. Parece existir uma relação estatisticamente significativa entre a área científica frequentada pelos respondentes e a priorização de doentes em função da capacidade benefício $\left(\mathrm{P}_{3}\right)$, da necessidade clínica $\left(\mathrm{P}_{4}\right)$ e da igualdade em saúde $\left(\mathrm{P}_{6}\right)$. 
TABELA 4 - Relação entre adesão aos princípios distributivos e as características sociodemográficas e de saúde dos respondentes, testes Mann-Whitney e Kruskal-Wallis

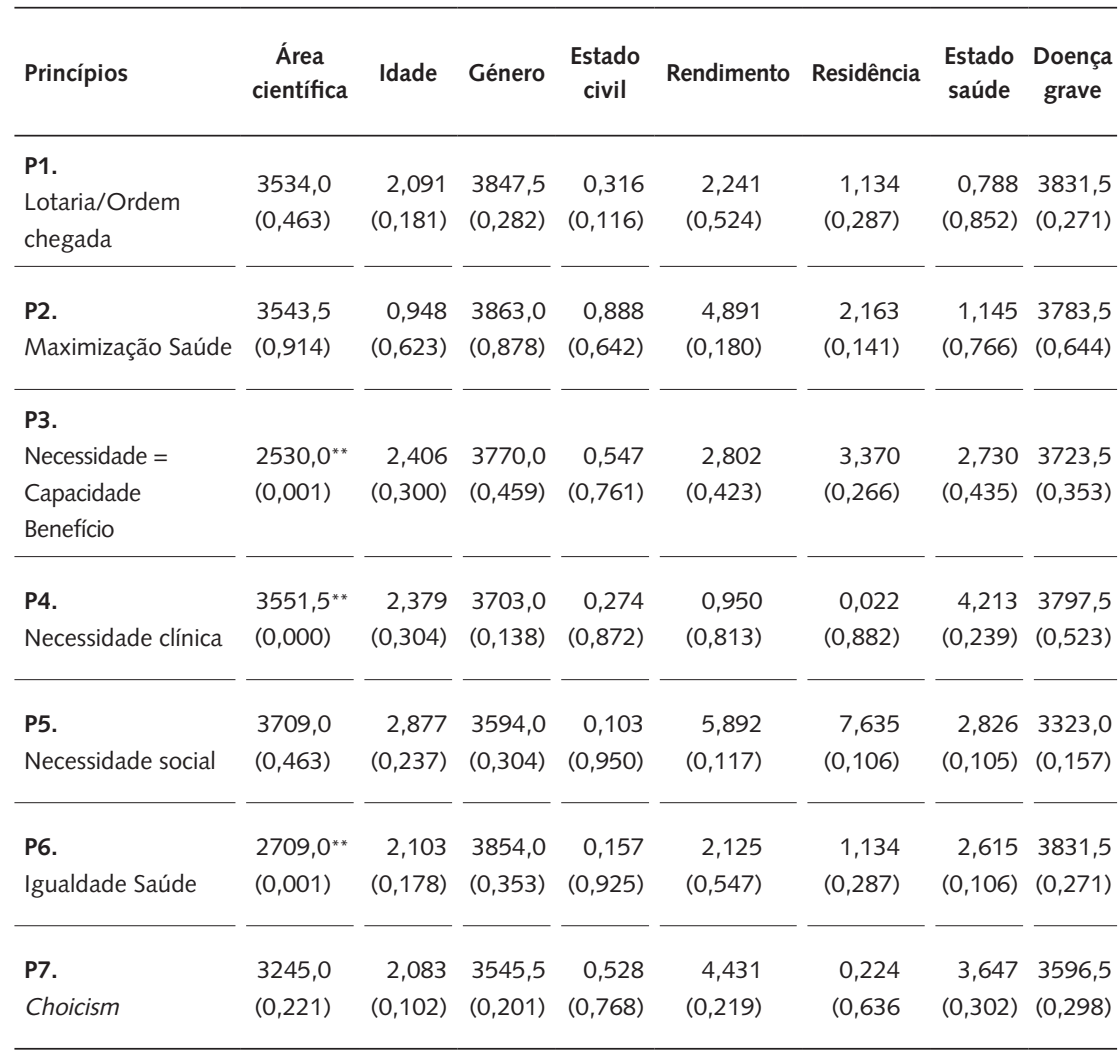

Nota: * *ível de significância de $p \leq 0.001$. Aplicou-se o teste Mann-Whitney para o grupo de variáveis: área científica, género, doença grave e residência. Para as restantes variáveis foi realizado o teste Kruskal-Wallis.

\section{Combinação de resultados quantitativos e qualitativos - Aderência dos princípios} distributivos à atribuição da primeira prioridade no acesso ao tratamento

Identificados os princípios distributivos através das justificativas mencionadas pelos respondentes, procuramos explorar a existência de alguma associação entre eles e a escolha do doente a tratar em primeiro lugar. Para o efeito, foi criada uma variável que traduz o doente que cada inquirido colocou em primeiro lugar de atendimento. A Tabela 5 apresenta os resultados da aplicação do teste não paramétrico Kruskal-Wallis, onde se assume na hipótese nula $\left(\mathrm{H}_{0}\right)$ que a escolha do primeiro doente a tratar não depende do princípio distributivo e, caso contrário, na hipótese alternativa $\left(\mathrm{H}_{1}\right)$. 
TABELA 5 - Princípios éticos determinantes na escolha do doente a ser tratado em primeiro lugar, teste Kruskal-Wallis

\begin{tabular}{|c|c|c|c|}
\hline Hipóteses & Descrição das hipóteses & $\begin{array}{l}\text { Teste qui- } \\
\text {-quadrado } \\
\text { (p-value) }\end{array}$ & Resultados \\
\hline$H_{1}$ & $\begin{array}{l}\text { A escolha do doente a ser tratado em } \\
\text { primeiro lugar depende do princípio } \\
\text { Lotaria/Ordem Chegada (P1) }\end{array}$ & $\begin{array}{r}3,576 \\
(0,129)\end{array}$ & $\begin{array}{l}\text { Não se rejeita a hipótese nula } \rightarrow \\
\text { a escolha do doente a ser tratado em } \\
\text { primeiro lugar não depende de } P_{1}\end{array}$ \\
\hline $\mathrm{H}_{2}$ & $\begin{array}{l}\text { A escolha do doente a ser tratado } \\
\text { em primeiro lugar depende do } \\
\text { princípio Maximização Saúde (P2) }\end{array}$ & $\begin{array}{l}18,010^{* *} \\
(0,000)\end{array}$ & $\begin{array}{l}\text { Rejeita-se a hipótese nula } \rightarrow \\
\text { a escolha do doente a ser tratado } \\
\text { em primeiro lugar depende de } P_{2}\end{array}$ \\
\hline $\mathrm{H}_{3}$ & $\begin{array}{l}\text { A escolha do doente a ser tratado } \\
\text { em primeiro lugar depende } \\
\text { do princípio Necessidade = } \\
\text { Capacidade Benefício (P3) }\end{array}$ & $\begin{array}{l}44,721^{* *} \\
(0,000)\end{array}$ & $\begin{array}{l}\text { Rejeita-se a hipótese nula } \rightarrow \\
\text { a escolha do doente a ser tratado } \\
\text { em primeiro lugar depende de } P_{3}\end{array}$ \\
\hline $\mathrm{H}_{4}$ & $\begin{array}{l}\text { A escolha do doente a ser tratado } \\
\text { em primeiro lugar depende do prin- } \\
\text { cípio Necessidade clínica (P4) }\end{array}$ & $\begin{array}{l}20,582^{* *} \\
(0,000)\end{array}$ & $\begin{array}{l}\text { Rejeita-se a hipótese nula } \rightarrow \\
\text { a escolha do doente a ser tratado } \\
\text { em primeiro lugar depende de } P_{4}\end{array}$ \\
\hline$H_{5}$ & $\begin{array}{l}\text { A escolha do doente a ser tratado } \\
\text { em primeiro lugar depende do } \\
\text { princípio Necessidade social (P5) }\end{array}$ & $\begin{array}{r}4,097 \\
(0,073)\end{array}$ & $\begin{array}{l}\text { Não se rejeita a hipótese nula } \rightarrow \\
\text { a escolha do doente a ser tratado } \\
\text { em primeiro lugar não depende de } \mathbf{P}_{5}\end{array}$ \\
\hline$H_{6}$ & $\begin{array}{l}\text { A escolha do doente a ser tratado } \\
\text { em primeiro lugar depende do } \\
\text { princípio Igualdade Saúde (P6) }\end{array}$ & $\begin{array}{l}15,271^{* *} \\
(0,000)\end{array}$ & $\begin{array}{l}\text { Rejeita-se a hipótese nula } \rightarrow \\
\text { a escolha do doente a ser tratado } \\
\text { em primeiro lugar depende de } \mathbf{P}_{6}\end{array}$ \\
\hline $\mathrm{H}_{7}$ & $\begin{array}{l}\text { A escolha do doente a ser tratado } \\
\text { em primeiro lugar depende do } \\
\text { princípio Choicism (P7) }\end{array}$ & $\begin{array}{r}0,296 \\
(0,587)\end{array}$ & $\begin{array}{l}\text { Não se rejeita a hipótese nula } \rightarrow \\
\text { a escolha do doente a ser tratado } \\
\text { em primeiro lugar não depende de } P_{7}\end{array}$ \\
\hline
\end{tabular}

Nota: **nível de significância de $p \leq 0.001$

Os resultados parecem indicar que a escolha do doente a atender em primeiro lugar teve múltiplas motivações associadas, nomeadamente a eficiência traduzida pela maximização dos ganhos em saúde e pela eficácia do tratamento, a necessidade clínica e a igualização da saúde ao longo da vida (fair-innings). Estes resultados convergem para os apresentados na Tabela 2 e na Tabela 3. Os resultados da Tabela 2 mostram que o doente Miguel foi maioritariamente ( $72 \%$ respondentes) escolhido para ser atendido em primeiro lugar. Por outro lado, as justificativas transcritas na Tabela 3 confirmam que os respondentes avançam razões de eficiência para escolher o Miguel, nomeadamente o benefício prolongar-se por mais tempo e o futuro papel contributivo do doente. A eminência de morte que o Miguel apresenta e o facto de ser o candidato mais novo aos tratamentos justificam 
a adesão ao princípio da regra do resgate e do fair-innings, respetivamente. A Tabela 2 mostra, ainda, que o Paulo foi o doente ao qual, a seguir ao Miguel, se atribuiu um grau máximo de prioridade no atendimento (23\% dos respondentes). Também para esta escolha do Paulo terá contribuído a maior eficácia do tratamento, a sua idade e o seu potencial papel contributivo.

\section{Discussão e conclusão}

Racionar cuidados de saúde tem-se revelado um assunto complexo e controverso, sobretudo a nível micro, onde escolhas obrigam a selecionar doentes. Definir os critérios que devem suportar o estabelecimento de prioridades entre doentes é uma tarefa que, embora longe de estar cumprida, importa concluir. A urgência na definição destes critérios é acentuada com a possibilidade de numa sociedade existirem julgamentos de valor conflituantes entre os principais atores sociais.

O presente estudo exploratório procura contribuir para este debate, ao analisar as preferências de dois potenciais atores da sociedade portuguesa que, salvaguardadas as devidas distâncias por falta de experiência profissional, poderão vir a assumir um envolvimento direto neste tipo de decisões. Com recurso a uma abordagem quali-quantitativa, foi explorado o parecer de dois grupos de estudantes universitários, com formações em ciências empresariais e em ciências da saúde, quanto à ordenação de doentes com características pessoais e condições de saúde distintas.

Os resultados do estudo revelaram que os respondentes não só não evitaram priorizar os doentes, como aderem a um pluralismo de princípios distributivos, em conformidade com a evidência empírica (Cookson e Dolan, 1999; Exel et al., 2015). Os dois grupos de estudantes entendem que os doentes devem ser priorizados de acordo com a: 1) equidade intergeracional - fair-innings; 2) gravidade do estado de saúde e 3) maximização dos ganhos esperados em saúde. Ainda que o padrão de preferências pela ordenação dos doentes seja globalmente semelhante entre os dois grupos de estudantes, existe alguma divergência no que respeita à adesão ao argumento do fair-innings ou ao suporte da equidade intergeracional. Os estudantes das ciências da saúde parecem definir prioridades de atendimento em razão direta com a eficácia clínica dos tratamentos. Simultaneamente, parecem valorar menos a idade, por si só, do que os demais respondentes. Por esta razão, revelam uma menor propensão a conceder prioridade aos mais novos apenas pela idade. Estas preferências manifestadas pelos estudantes das ciências da saúde convergem para os resultados internacionais que avaliaram os pareceres de profissionais de saúde (Antiel et al., 2013; Johri et al., 2005; Lees et al., 2002; Strech et al., 2009; Winkelhage et al., 2013). Estes resultados parecem sugerir que os 
futuros profissionais de saúde, em Portugal, poderão estar bastante mais sensibilizados para a prossecução do critério da eficiência, avaliado em termos de ganhos de saúde, do que se poderia imaginar.

Os nossos resultados revelam ainda, em conformidade com os resultados ingleses (Cookson e Dolan, 1999), mas contrariamente à maior parte dos dados da evidência empírica internacional (Kasemsup et al., 2008; Exel et al., 2015), que os dois grupos de estudantes inquiridos não parecem penalizar os fatores endógenos da doença, refletidos nos estilos de vida. No entanto, não deixa de ser curioso que a doente Fernanda tenha sido colocada em última e penúltima prioridade de atendimento pelos dois grupos de estudantes. Uma possível explicação para esta ordenação pode estar no facto de os doentes serem descritos por várias características. Excluindo o fator estilo de vida da Fernanda, ela é uma doente de meia-idade que não está nem em grande sofrimento, nem em risco de se tornar dependente de outros.

Em termos gerais, podemos concluir que os dois grupos de estudantes inquiridos não parecem ter uma visão ética muito discordante. Adicionalmente e, salvaguardadas as diferenças metodológicas entre o nosso estudo e o inglês, parece que os julgamentos de valor dos estudantes portugueses são semelhantes aos da população inglesa. Poderá, no entanto, existir uma tensão quanto à prioridade conferida às crianças, sobretudo se os tratamentos que lhes são destinados forem pouco eficazes. Assim, parece que os estudantes das ciências sociais têm uma visão semelhante aos estudantes das ciências económicas e aos membros da população inglesa, embora confiram menor prioridade aos doentes mais novos. A eventual diferença de opinião entre os dois grupos inquiridos levanta questões interessantes sobre o papel que deve ter a participação da população na tomada de decisões na saúde. Se acrescentarmos o facto de os respondentes não se terem escusado de priorizar os doentes, poderemos ter reunidas as condições para desenvolver, em Portugal, um processo de reflexão sobre a possibilidade de implementar um processo explícito de racionamento que conceba uma sistematização e transparência dos critérios de priorização em saúde. Esta ideia parece encontrar eco na estratégia relativa à cidadania e saúde prevista no Plano Nacional de Saúde, atualmente em vigor (PNS, 2012-2016). Esta estratégia realça o papel decisório da sociedade ao colocar o "lugar do cidadão" nos sistemas de saúde e na implementação de políticas e programas que visem ganhos em saúde (ibidem). Neste sentido, o cidadão assume um papel decisório na saúde individual, na saúde coletiva, na organização dos sistemas e na definição de programas e prioridades.

Estes resultados devem ser analisados com a cautela intrínseca a um estudo exploratório, dadas as limitações da amostra. A amostra, para além de reduzida, é composta apenas por estudantes universitários, não sendo por isso 
representativa dos atores sociais que pretende representar. Adicionalmente, a composição da amostra denota um certo enviesamento, dada a diferença numérica existente entre o grupo dos estudantes das ciências económicas e das ciências da saúde. Para além das limitações associadas à amostra existe, ainda, o inconveniente relacionado com a análise qualitativa, nomeadamente o sistema de contagem. É reconhecido que o número de vezes que um fenómeno particular é mencionado não está, necessariamente, correlacionado com a sua significância social. Neste contexto, o facto de um respondente não ter aludido a um determinado princípio aquando da sua justificação da escolha do doente não nos permite concluir com certeza que discorde dele. Acreditamos que estas limitações são compensadas pelo contributo deste estudo.

Os resultados encontrados poderão constituir um importante indicador político, na medida em que parecem denunciar que qualquer processo de microalocação de recursos que venha a ser desenvolvido em Portugal deve, para ser socialmente legitimado, assumir uma tipologia mista que incorpore considerações técnicas, traduzidas pela procura da maximização dos ganhos de saúde e equitativas, que reflitam considerações de igualdade intergeracional e da gravidade do estado de saúde.

Futuras investigações seriam interessantes se esta metodologia fosse aplicada a uma amostra representativa de profissionais de saúde e de administradores hospitalares. Adicionalmente, seria oportuno aplicar um estudo idêntico à população, em geral, para averiguar quais são os princípios distributivos aceites num processo de racionamento ao nível micro e compará-los com a visão dos outros dois atores sociais (profissionais de saúde e administradores hospitalares).

\section{Referências bibliográficas}

Antiel, Ryan; Curlin, Farr; James, Katherine; Tilburt, Jon (2013), "The Moral Psychology of Rationing among Physicians: The Role of Harm and Fairness Intuitions in Physician Objections to Cost-effectiveness and Cost-containment”, Philosophy, Ethics and Humanities in Medicine, 8, 8-13.

Bardin, Laurence (2013), Análise de conteúdo. Lisboa: Edições 70.

Beauchamp, Tom; Childress, James (2001), Principles of Biomedical Ethics. New York: Oxford University Press [5. ${ }^{a}$ ed.].

Broome, John (1984), "Selecting People Randomly", Ethics, 95(1), 38-55.

Butler, John (1999), The Ethics of Health Care Rationing: Principles and Practices. London: Cassell.

Childress, James (1983), "Who Shall Live when Not All Can Live", in Ronald Munson, Intervention and Reflection: Basic Issues in Medical Ethics. California: Wadsworth 
Publishing Company, 497-505. Versão original publicada em Soundings (1970), 53, 339-355.

Clark, Sarah; Weale, Albert (2012), "Social Values in Health Priority Setting: A Conceptual Framework", Journal of Health Organization and Management, 26(3), 293-316.

CNECV - Conselho Nacional Ética para as Ciências da Vida (2012), Parecer sobre um modelo de deliberação para financiamento do custo dos medicamentos. 64/ CNECV/2012. Lisboa.

Coast, Joanna (2000), "Explicit Rationing, Deprivation, Disutility and Denial Disutility: Evidence from a Qualitative Study", in Angela Coulter; Chris Ham (orgs.), The Global Challenge of Health Care Rationing. Buckingham: Open University Press. Milton Keynes, 192-200.

Cookson, Richard; Dolan, Paul (1999), "Public Views on Health Care Rationing: A Group Discussion Study”, Health Policy, 49(1-2), 63-74.

Constituição da República Portuguesa (2005), Coimbra: Almedina.

Culyer, Anthony (1997), "The Principle Objective of the NHS Should Be to Maximize Aggregate Health", in Bill New (org.), Rationing: Talk and Action in Health Care. London: King's Fund and British Medical Journal Publishing Group, 95-100.

Culyer, Anthony; Wagstaff, Adam (1993), "Equity and Equality in Health and Health Care", Journal of Health Economics, 12(4), 431-457.

Decreto-Lei n. ${ }^{\circ}$ 233/2005 de 29 dezembro. Diário da República n. 249 - I Série-A. Ministério da Saúde. Lisboa.

Diederich, Adele; Salzmann, Daniela (2015), "Public Preferences Regarding Therapeutic Benefit, Costs of a Medical Treatment and Evidence-based Medicine as Prioritization Criteria”, Journal of Public Health, 23(3), 1-12.

Dolan, Paul; Cookson, Richard; Ferguson, Brian (1999), "Effect of Discussion and Deliberation on the Public's Views of Priority Setting in Health Care: Focus Group Study", British Medical Journal, 3318(7188), 916-919.

Exel, Job; Baker, Rachel; Mason, Helen; Donaldson, Cam; Brouwer, Werner (2015), "Public Views on Principles for Health Care Priority Setting: Findings of a European Cross-country Study Using Q Methodology”, Social Science \& Medicine, 126, 128-137.

Fortes, Paulo; Zoboli, Elma (2002), "A Study on the Ethics of Microallocation of Scarce Resources in Health Care”, Journal of Medical Ethics, 28(4), 266-300.

Gagnon, Marie-Pierre; Desmartis, Marie; Lepage-Savary, Dolorès; Gagnon, Johanne; St-Pierre, Michèle; Rhainds, Marc; Lemieux, Renald; Gauvin, Francois-Pierre; Pollender, Hugo; Légaré, France (2011), "Introducing Patients' and the Public's Perspectives to Health Technology Assessment: A Systematic Review of International Experiences”, International Journal of Technology Assessment in Health Care, 27(1), 31-42.

Hadorn, David (1991), "Setting Health Care Priorities in Oregon. Cost Effectiveness Meets the Rule of Rescue”, Journal of American Medical Association, 265(17), 2218-2225. 
Johri, Mira; Damschroder, Laura; Zikmund-Fisher, Brian; Ubel, Peter (2005), “The Importance of Age in Allocating Health Care Resources: Does Intervention-type Matter?", Health Economics, 14(7), 669-678.

Kahneman, Daniel; Tversky, Amos (1979), "Prospect Theory: An Analysis of Decision under Risk”, Econometrica, 47, 263-291.

Kaplan, Giora; Baron-Epel, Orna (2013), “The Public's Priorities in Health Services”, Health Expectations, 18(5), 904-917.

Kasemsup, Vijj; Schommer, John; Cline, Richard; Hadsall, Ronald (2008), “Citizen's Preferences Regarding Principles to Guide Health Care Allocation Decisions in Thailand", Value in Health, 11(7), 1194-1202.

Lees, Ann; Scott, Nicholas; Scott, Sheila; MacDonald, Sara; Campbell, Christine (2002), "Deciding How NHS Money is Spent: A Survey of General Public and Medical Views", Health Expectations, 5(1), 47-54.

LeGrand, Julian (1982), The Strategy of Equality. London: Allen and Unwin.

Mooney, Gavin (1994), Key Issues in Health Economics. New York: Harvester Weatsheaf.

Persad, Govind; Wertheimer, Alan; Emanuel, Ezekiel (2009), "Principles for Allocation of Scarce Medical Interventions", Lancet, 373(9661), 423-431.

Pinho, Micaela (2014), "The Dilemma of Managing Scarce Health Care Resources: Evidence of the Conflict between Economic or Ethical Principles in Microallocation Decisions”, International Journal of Management Science and Information Technology, 14(14), 1-11.

PNS - Plano Nacional de Saúde - 2012-2016. Consultado a 10.12.2015, em http://pns. dgs.pt.

Powers, Madison; Faden Ruth (2008), Social Justice: The Moral Foundations of Public Health and Health Policy. New York: Oxford University Press.

Rabin, Matthew (1998), "Psychology and Economics", Journal of Economic Literature, 36(1), 11-46.

Rawls, John (1999), A Theory of Justice. Oxford: Oxford University Press.

Reeves, Aaron; McKee, Martin (2015), "The Attack on Universal Health Coverage in Europe: Recession, Austerity and Unmet Needs”, European Journal of Public Health, 25(3), 364-365.

Reys, Lesseps (1991), "A alocação de recursos médicos nas unidades de cuidados intensivos”, Ata Médica Portuguesa, 4, 23-26.

Roemer, John (1998), Equality of Opportunity. Cambridge Mass: Harvard University Press. Sen, Amartya (1980), "Equality of What?", in The Tanner Lectures on Human Values.

Volume I. Cambridge: Cambridge University Press, 197-220.

Strech, Daniel; Persead, Govin; Marckmann, George; Danis Marion (2009), “Are Physicians Willing to Ration Health Care? Conflicting Findings in a Systematic Review of Survey Research", Health Policy, 90(2-3), 113-124.

Williams, Alan (1997), "Intergenerational Equity: An Exploration of the 'Fair Innings' Argument”, Health Economics, 6(2), 117-132. 
Williams, Alan; Cookson, Richard (2000), "Equity in Health”, in Anthony J. Culyer; Joseph P. Newhouse (orgs.), Handbook of Health Economics. Amsterdam, North-Holland: Elsevier, 1863-1907.

Winkelhage, Jeannette; Schreier, Margrit; Diederich, Adele (2013), "Priority Setting in

Health Care: Attitudes of Physicians and Patients”, Health, 5(4), 712-719.

Artigo recebido a 04.06.2016

Aprovado para publicação a 13.03.2017

\section{Micaela Pinho}

Universidade Portucalense, Research on Economics, Management and Information Technologies REMIT, Instituto Jurídico Portucalense - IJP

Rua Dr. António Bernardino de Almeida, 541, 4200-072 Porto, Portugal

Contacto: michaelapinho@hotmail.com

\section{Ana Pinto Borges}

Instituto Superior de Administração e Gestão, European Business School

Rua de Salazares, 842, 4100-442 Porto, Portugal

Contacto: anapintoborges@hotmail.com

\section{Which Ethical Principles Should Define Prioritization of Patients?}

This article aims to study and compare the value judgments of Portuguese college students from the business and health fields regarding patient prioritization and compare these value judgments indirectly with those from the general public in England. For the study, we utilized a qualitative and quantitative approach applied to a hypothetical rationing exercise involving the prioritization of four patients, distinguished by their personal characteristics and health conditions. Data were analyzed through bivariate and content analysis. We concluded that Portuguese students support a plurality of ethical principles similar to those held by English society. However, there may be a conflict of opinions among

\section{Quels sont les principes éthiques qui doivent définir la mise en place de priorités entre les malades?}

Cet article a pour but de collecter et de comparer les jugements de valeur d'étudiants universitaires portugais du domaine entrepreneurial et de la santé en matière de priorisation de malades et de comparer ces jugements, indirectement, à ceux de la population anglaise. Pour ce faire, nous avons fait appel à une approche qualitative et quantitative appliquée à un exercice hypothétique de rationnement qui entraîne la priorisation de quatre patients, différenciés par leurs caractéristiques personnelles et leurs états de santé. Les données ont été traitées par le biais d'analyses bivariées et de contenu. Nous sommes parvenus à la conclusion que les étudiants portugais adoptent un 
Portuguese students regarding the prioritization of younger patients.

Keywords: ethical principles; healthcare; hospital management; patients' prioritization; Portugal. pluralisme de principes éthiques semblable à ceux de la société anglaise. Néanmoins, il peut exister chez les étudiants portugais un conflit d'opinions pour ce qui a trait à la priorisation des patients les plus jeunes.

Mots-clés: gestion hospitalière; priorisation des patients; Portugal; principes éthiques; soins de santé. 